\title{
Theoferometer for the Construction of Precision Optomechanical Assemblies
}

\author{
Ashley M. Korzun ${ }^{*}$ \\ University of Maryland, College Park, MD, 20742
}

\begin{abstract}
The increasing difficulty of metrology requirements on projects involving optics and the alignment of instrumentation on spacecraft has reached a turning point. Requirements as low as $\mathbf{0 . 1}$ arcseconds for the static, rotational alignment of components within a coordinate system cannot be met with a theodolite, the alignment tool currently in use. A "theoferometer: is an interferometer mounted on a rotation stage with degrees of freedom in azimuth and elevation for metrology and alignment applications. The success of a prototype theoferometer in approaching these metrology requirements led to a redesign stressing mechanical, optical, and software changes to increase the sensitivity and portability of the unit. This paper covers the improvements made to the first prototype theoferometer, characteristic testing, and demonstration of the redesigned theoferometer's capabilities as a "theodolite replacement" and low-uncertainty metrology tool.
\end{abstract}

\section{Introduction}

$\mathrm{C}$ oordinate and vector metrology is invaluable to the assembly and alignment of spacecraft, flight hardware, and optical test beds. Mirrors, sensors, benches, and detectors form the critical components of most satellites, telescopes, and control systems, and their successful operation is heavily dependent on the tolerance of the instruments used in their alignment. The envelope of these metrology measurements drives the science and performance objectives of many current and future projects.

The current, accepted tool for vector and coordinate metrology is the theodolite. Essentially a lighted telescope, the theodolite sends out a collimated reticule image, which is reflected off of a mirrored alignment cube and back into the telescope, producing a second set of reticules. By eye, the user can adjust the orientation of the theodolite in azimuth and elevation to overlap the reticules, where elevation is measured with respect to the local gravity vector. This position, with the reticules aligned, gives the direction of the vector normal to the surface of the mirrored cube face. Using a system of theodolites and several mirrored alignment cubes, the faces of several cubes are related to a primary reference cube, and this establishes a coordinate system for the aligned hardware. A more detailed description of the methods of vector metrology is beyond the scope of this paper and can be found under the provided references. $^{1,2}$

The measurement uncertainty of the theodolite is driven by the difficulty of repeating measurements, vibration of the theodolite and test surface, limitations in the knowledge of the local gravity vector, and the ability of the human eye to perfectly align the reticules with the telescope. With an experienced user, these errors add up to a typical measurement uncertainty of \pm 2 arcseconds for a single measurement. With this uncertainty characteristic, the theodolite is incapable of meeting current and future project requirements of sub-arcsecond metrology.

Named for its use of interferometry and theodolite capability, the theoferometer is an instrument developed to have a sub-arsecond measurement uncertainty by reducing or eliminating the error sources inherent in the theodolite. It consists of a Twyman-Green interferometer mounted on a 2-axis rotation stage with piezoelectric motors actuating position adjustment and high accuracy optical encoders providing position readout. A discussion of the prototype theoferometer hardware and software, characteristic testing, results, and future work follows.

\section{Optomechanical Design}

The theoferometer has three main hardware components: an optical stage, an azimuth stage, and an elevation stage. The optical stage of the theoferometer is an unequal path interferometer (see Figure 1). Following the light path, a beam originates from a He-Ne laser. After being spatially filtered, expanded, and collimated, the beam passes

${ }^{*}$ Undergraduate, Department of Aerospace Engineering, 3181 Martin Hall, student member. 
through a beamsplitter, where it is divided into two beams, with one onto the test surface and the other directed onto a mirrored reference cube. The two beams return along the same path and recombine at the beamsplitter and are folded $90^{\circ}$ into a charge-coupled device (CCD) image plane.

The interference patterns appearing on the CCD display are collected by the user with a frame grabber package and saved as bitmap files. A typical fringe pattern is shown in Figure 2. These fringe patterns are processed using an Fast Fourier Transform algorithm written in software custom-built for in Interactive Data Language (IDL). The software provides output of the physical orientation of the test surface as compared to the reference cube from the fringe tilt passed to the routine as a bitmap image. The fringe processing software is discussed further in Section III.

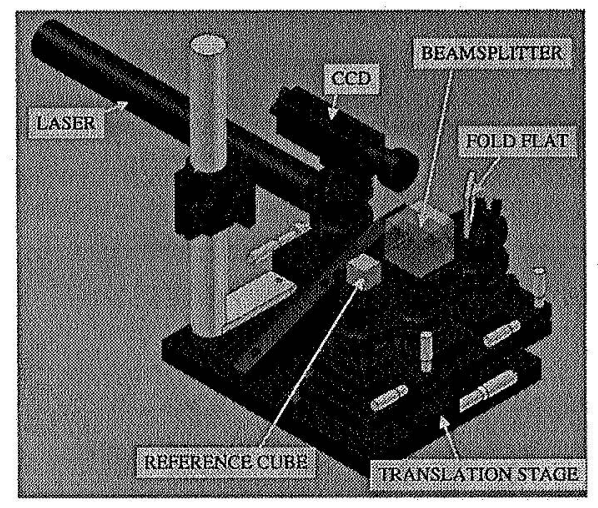

The optical stage is mounted on a 2-axis rotation platform, with decoupled motion in azimuth and elevation. Separate azimuth and elevation stages are fixed to nearlyfrictionless air bearings which allow $360^{\circ}$ of rotation in azimuth and approximately $25^{\circ}$ in elevation. Stage rotation can be induced coarsely, by hand adjustment, or finely, using piezoelectric linear inchworm motors actuated against an arm clamped to the bearing assembly. The motors can produce rotations as fine as 0.06 arcseconds.

The optical encoders, developed at NASA/GSFC, are critical to the sub-

Figure 1. Optical Stage Layout arcsecond measurement uncertainty level

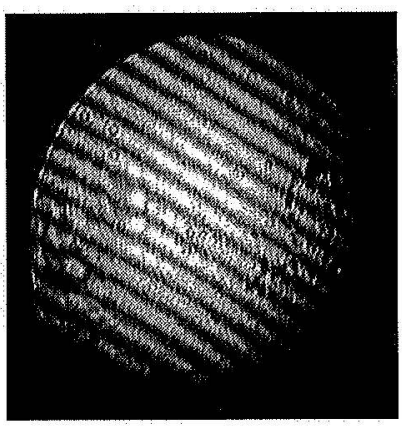

Figure 2. Typical Fringe Pattern

goal for the theoferometer. A silicon disk containing patterns of code bits and fiducials (see Figure 3 ) is mounted to the central shaft of the air bearing. An LED flashes behind the disk, illuminating an image which is captured by a CCD readhead on the front side of the encoder disk, sending the image to the computer. Figure 4 shows the encoder disk / readhead assembly.

The encoder software uses the spacing of the fiducials to determine the angular position of the theoferometer with a resolution of 0.04 arcseconds. An encoder / readhead assembly is mounted on both azimuth and elevation stages. A second readhead is positioned $180^{\circ}$ from the first to protect against runout error of the encoder disk. Any error will be equal in magnitude, but opposite in sign, and the software eliminates this error in the position calculation by using readings from each readhead.

The combination of position readings from the encoders and fringe pattern tilts from the IDL software allows the user to take orientation readings in azimuth and elevation akin to those taken with a theodolite. However, with the theoferometer, the theodolite-equivalent null fringes cannot be processed, so the physical tilts provided by the IDL software are actually how far away from null the fringes are, and signs can be assigned to the tilt directions. The theoferometer-equivalent metrology measurements are an algebraic sum of the position and tilt readings.

\section{Fringe Processing Software}

An important design characteristic of the theoferometer is the associated fringe processing software. The theoferometer delivers position measurements anti-parallel to a test surface by checking a position away from null, the desired position, and backing out how far away from null the system is using a custom fringe processing software package. This software links properly oriented tilt fringes to the physical orientation of a test surface by Fourier analysis and built in IDL functions. Properly oriented fringes are those oriented left-to-right, or in the $2^{\text {nd }}$ and $4^{\text {th }}$ quadrants. A more detailed description of the algorithm is covered in Reference 4.

The interferograms, saved as bitmaps, are first decomposed into their component spatial frequencies. For a black and white fringe pattern, there are only two spatial frequencies: horizontal and vertical. The number and orientation 
of the fringes is proportional to the physical tilt between the reference surface and a test surface, and so the measurement of the spatial frequency is also a measurement of this relative tilt. Once decomposed, with a Hanning window applied, and the image zero padded, the image is Fourier transformed. Then, the power spectrum is calculated for the image and used for the remainder of the algorithm.

The central spike in the power spectrum, a result of background illumination, is eliminated and a threshold to reduce noise is applied to the array. From the remaining image array, the software selects a subarray around the next greatest peak and zeroes out everything else. This peak is treated as a Gaussian and the center of the peak is determined. The spatial frequency is indicated by this peak and is a function of the distance from the peak to the zero frequency elements of the array and the size of the array dimension.

To relate the spatial frequency to the physical tilt, the tilt between the two surfaces is treated as an air wedge creating Fizeau-type fringes. The inverse of the space between the fringes gives the spatial frequency in metric units. The wedge half-angle $\alpha$ is a function of the wavelength, spatial frequency, number of pixels, and the aperture size. With the aperture size determined from the diameter of the incident laser beam, this wedge half-angle is also the tilt angle between the reference cube and the test surface. The proper sign of the fringes is given by the whether the half-angle is above null, or below null. Preliminary testing shows the software to be most sensitive for $12-15$ fringes per shot and processed tilt values under 50 arcseconds.

\section{Experimental Setups and Procedures}

The following section describes the three experimental setups and procedures used to align and characterize the capability of the theoferometer as a low uncertainty metrology tool. The testing covered is leveling the azimuth stage, adjusting the orthogonality of the azimuth and elevation stage assembly, and the sensitivity of the theoferometer in detecting surface tilts. The sensitivity testing includes the software fringe analysis and entire theoferometer system. The results from each experiment are discussed in Section V.

\section{A. Azimuth Stage Leveling}

Unlike a theodolite, the theoferometer has no common reference point, which is typically an internal gravity reference. As a result, the azimuth stage of the theoferometer must be adjusted from its assembled position to one normal to the local gravity vector. Using gravity to set the position of the azimuth stage establishes a standard for recreating lab setups and taking measurements. It also allows for all data collected with the theoferometer to have the same reference as data taken with a theodolite.

The horizontal center of the meniscus of any liquid will always be normal to the local gravity vector. This property makes the mercury level, an enclosed pool of mercury with adjustable legs, a suitable external gravity reference for leveling the theoferometer's azimuth stage. The reflective nature of liquid mercury allows for a return off the surface, much like a liquid mirror. The adjustable legs of the mercury level's stand tilt the surface of the level such that the return of the beam is off the meniscus and not the protective glass of the level.

The lab setup (see Figure 5) is built on a granite tabletop, first in the position shown. Following the light path in the figure, the portion of the beam leaves the optical stage through the beamsplitter. The light is folded $90^{\circ}$ through a pentaprism and onto the meniscus of the mercury level, where it is reflected and returns to the optical stage along the same path. The beam returning from the reference cube on the optical stage and the beam returning from the mercury level are out of phase, and the resulting interference fringe pattern is folded into the CCD and visible on the user interface.

A bubble level and shims were used to level the pentaprism, ensuring the beam was turned $90^{\circ}$ from the position it returned off the mercury level. Shallow interference fringes were visible when the mercury level was placed flat on the tabletop, but moving the theoferometer did nothing to change the orientation, density, or contrast of the fringes, indicating the visible fringes with the level in a flat position were from the reflection off the protective glass of the level. Adjusting the legs on the mercury level to tilt the glass surface a few degrees was substantial enough to prevent the return off the glass from entering the pentaprism and allow the primary return off the meniscus of the mercury level to enter the pentaprism.and recombine with the reference beam on the theoferometer.

The fringes off the mercury pool were sharp in contrast and responded to position changes of the theoferometer, unlike the fringes off the protective glass. Small vibrations from hardware on the table permeated through the mercury pool, and the vibrations were seen in the fringes, indicating the visible return was off the surface of the liquid and not the glass. Altering the position of the mercury level enough to move the return off the horizontal 
center of the meniscus caused significant distortion to the fringe patterns and deviated the return beam away from

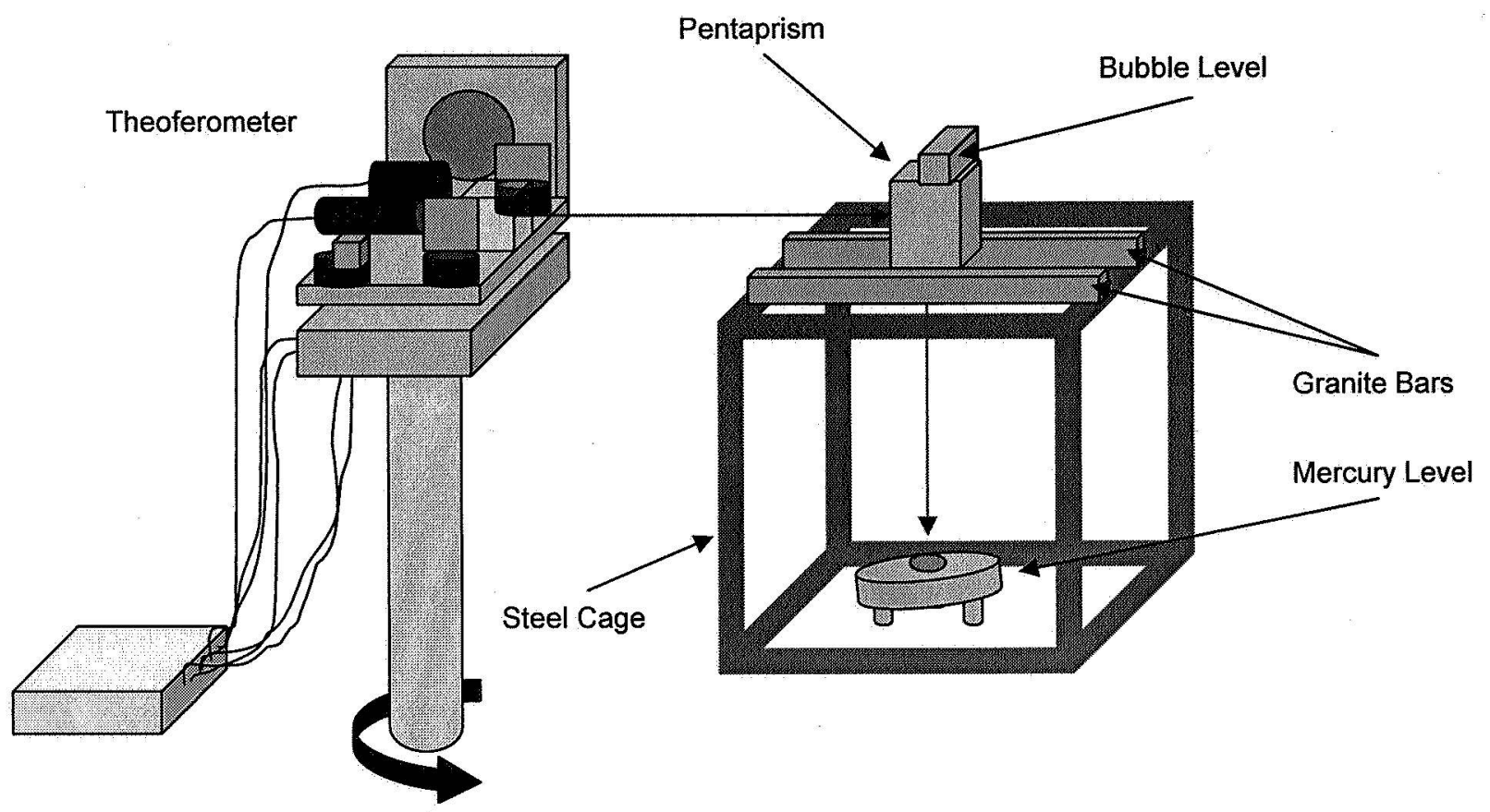

Figure 5. Azimuth Stage Leveling Experimental Setup

the pentaprism. The leveling of the pentaprism and a return though the window in the base of the pentaprism off the mercury pool indicated the return was coming from the horizontal center of the meniscus of the mercury pool. The gravity isolation function of the tabletop was not used. While the setup would be isolated from environmental vibration, the floating table would prevent the gravity vector from remaining in a constant direction.

Position 1 is the starting position of the theoferometer and associated experimental setup, and 10 shots of the fringe patterns were taken from the user interface in this position. Using readings from the encoders, the theoferometer was rotated $90^{\circ}$ counterclockwise and relocked, and the entire setup was moved $90^{\circ}$ as well. Ten more shots were taken at Position 2, and the process repeated until position readings and shots were taken at 4 positions, each $90^{\circ}$ apart. The 40 fringe patterns, 10 shots from each of 4 positions, were processed using the IDL fringe analysis software, and the tilt values and encoder position readings were stored in a data file for later analysis.

\section{B. Stage Orthogonality}

This test checks the orthogonality of the azimuth and elevation stages to a higher degree than can be done with a level during the assembly of the theoferometer. If the two stages are not orthogonal to one another, position changes in elevation will cause an angular displacement, or runout error, in the azimuth direction. This unexpected azimuth displacement leads to additional error in measurements being compared across varying elevation positions.

Figure 6 shows the experimental setup for the adjustment of stage orthogonality. Following the light path, a part of the beam exits the optical stage and is directed onto a dihedral, or two flat mirrors bonded at a $90^{\circ}$ angle. After striking one of the mirrors of the dihedral, the beam is reflected off the other mirror and returns to the beamsplitter on the theoferometer along the same path. The beams from the dihedral and reference cube are out of phase, and the resulting interference fringes are folded into the $\mathrm{CCD}$ and visible on the user interface.

Beginning with the setup at as low an elevation reading as possible to return the beam back into the beamsplitter and subsequently recombine with the reference beam into the CCD, 10 shots were taken at this first elevation position. Using the encoder elevation position readings, the theoferometer was manually adjusted to increase the elevation position of the optical stage by approximately $1^{\circ}$, and another 10 shots were taken at this new position. The maximum elevation range provided by the theoferometer in this setup is $6.5^{\circ}$, which was large enough to detect the runout error evident as displacement in the azimuth readings. The fringe shots were processed using the IDL fringe analysis software, and the calculated tilt values were stored in a data file for later analysis. 


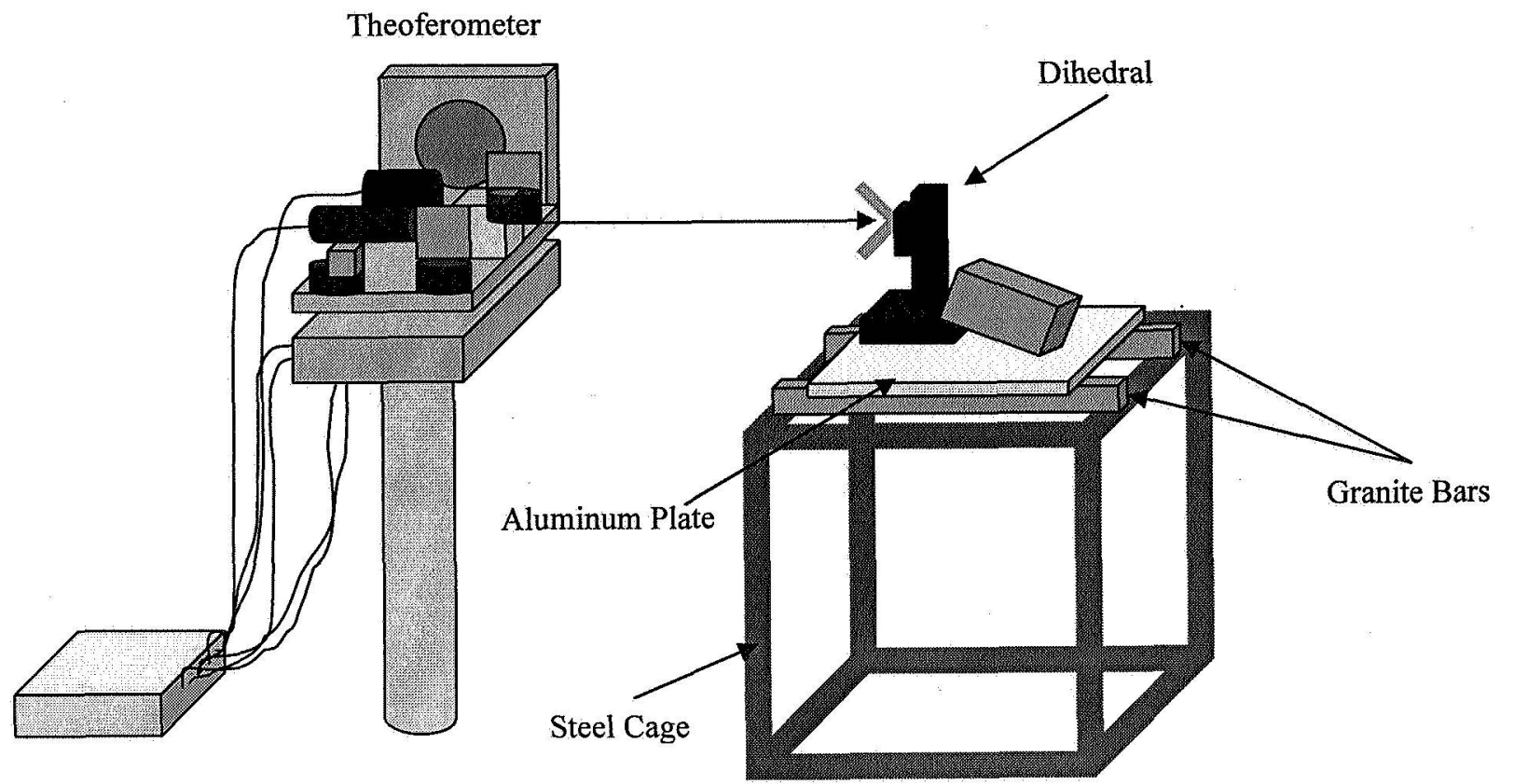

Figure 6. Stage Orthogonality Experimental Setup

\section{Sensitivity / Characterization of Measurement Uncertainty}

The measurement uncertainty of the theoferometer is characterized through sensitivity testing. This includes checking the software fringe analysis against induced tilts in a test surface and the theoferometer's ability to generate position readings for metrology applications at the sub-arcsecond level. This also begins to quantify the effects of vibration, stability, and mechanical drift on the measurement capability.

Checking the software sensitivity and the metrology measurement uncertainty of the theoferometer use the same experimental setup (see Figure 7). Following the light path, a part of the laser beam leaves the optical stage of the theoferometer and is folded $90^{\circ}$ through a pentaprism and then, another $90^{\circ}$ through a fold flat onto the mirror of a small angle generator. The small angle generator is an instrument calibrated to 0.01 arcseconds over a range of 200 arcseconds, where sub-arcsecond tilts are induce to a flat mirror. ${ }^{1}$ Micrometers push on sine bars attached to the mirror, producing very small and predictable changes to the orientation of the mirror about one or two axes (azimuth and/or elevation), making the small angle generator the best option for verifying physical tilts.

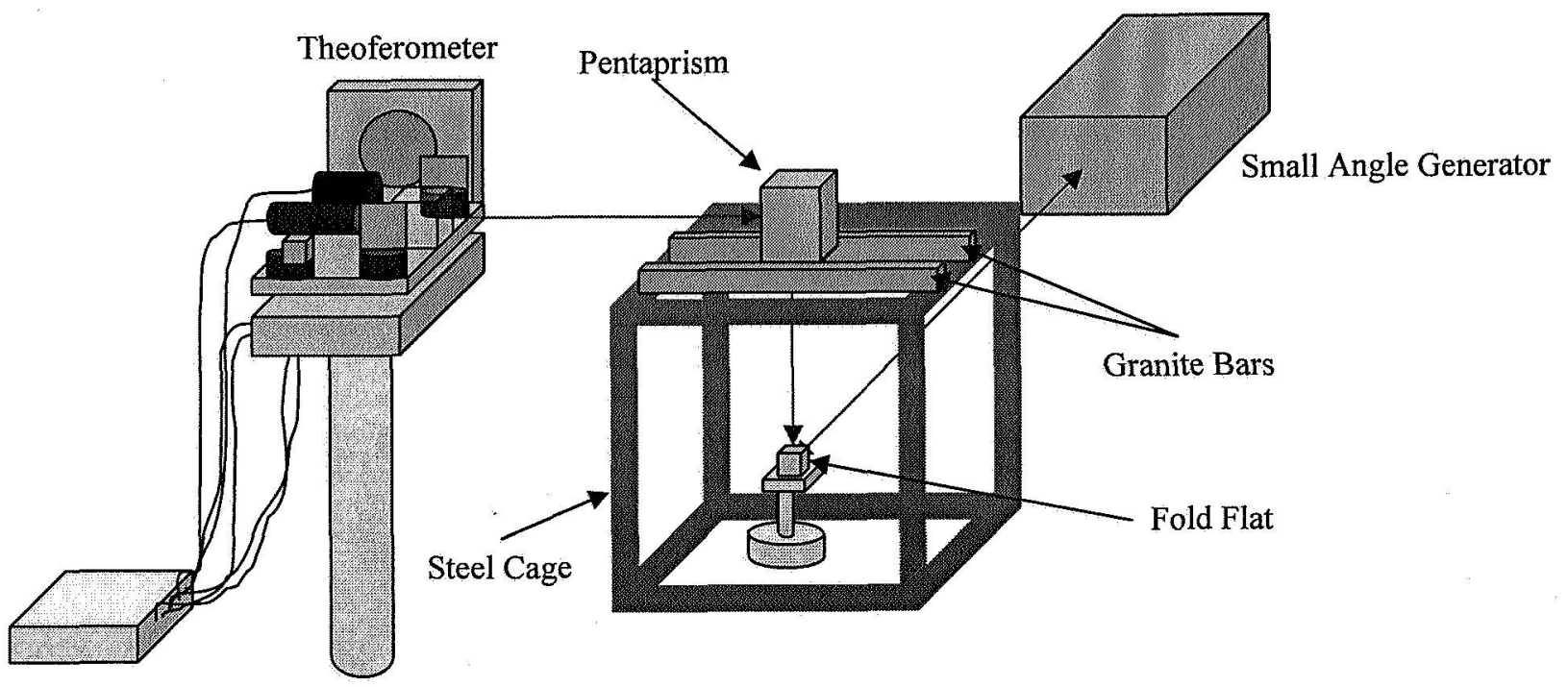

Figure 7. Sensitivity Testing Setup 
To start, the micrometers were set to zero in both azimuth and elevation, and the fold flat and pentaprism adjusted until a null fringe pattern was visible in the user interface display, indicating the incoming beam is perfectly normal to the mirror surface. The orientation of the mirror was then set to be approximately $30^{\circ}$ away from null in azimuth and approximately $40^{\circ}$ away from null in elevation, where null is zero tilt induced to the mirror. After taking 10 images, or shots, with the theoferometer of the interference fringes from the user interface, the micrometers were moved in increments of -1 arcsecond and another 10 shots taken at each position over a range of 25 arcseconds. The 250 fringe patterns, from 10 shots at each of 25 induced mirror tilt positions, were processed by the IDL fringe analysis software, with the resulting tilt values stored in a text file for later analysis.

\section{Experimental Results}

All data collected was for encoder position readings and tilts from the fringe processing software. With the exception of the software sensitivity data, all analysis was done using corrected position readings. Once the reticules on the theodolite are aligned, the position readings are at null, or give the position normal to the test surface. The theoferometer cannot process null "fringes", and instead, it uses positions away from null. The induced tilt is backed out and used to find the actual null position and can be used as a direct comparison to theodolite measurement data.

Calculating true orientation readings of the test surface with respect to the reference cube on the theoferometer is dependent on the orientation, or sign, of the interference fringes. This sign is determined using the inchworm motors to actuate fine changes in the theoferometer's position and hence, visibly alter the orientation and density of the interference fringes. If increasing the number of fringes also increases the $\mathrm{Az}$ encoder readings, then the theoferometer has moved farther away from null, requiring the azimuth sign to be negative for the correct position reading at null. If increasing the number of fringes also increases the El encoder readings, then again, the theoferometer has moved farther away from null, requiring the elevation sign to be negative. Because of the required orientation of the fringes for the IDL fringe analysis software, the signs for azimuth and elevation are opposite.

\section{A. Azimuth Stage Leveling}

The azimuth stage leveling procedure involved checking for variations in elevation readings as the theoferometer was rotated through $360^{\circ}$ in azimuth. After the first round of data was taken from all 4 positions, the encoder readings were corrected by algebraically summing with the software tilt values. From the 10 data points at each of the 4 positions, the mean and standard deviation of the mean were found for each position as well as the mean and standard deviation of the mean across all 4 positions. The acceptable variation between elevation readings at the separate position readings was 4 arcseconds, using the mean values from each of the 4 positions. As long as this spread was greater than 4 arcseconds, adjustments were made manually to the three adjustment and locking screws on the base plate of the theoferometer, and the data retaken at all 4 positions. This iterative adjustment and checking process continued through several changes to the base plate until the mean corrected elevation readings from all 4 positions were within 4 arcseconds of one another. All three sets of screws were then stakes with epoxy to prevent this procedure from having to be repeated.

Figure 8 shows the mean elevation positions for each group of shots across a total of 13 adjustments to the azimuth stage. For a given position, the elevation data does not progressively increase or decrease, but rather reflect variations in adjusting one of three sets of adjustment and locking screws on the azimuth stage. The standard deviation of the mean across all 4 positions after the final adjustment is 2.28 arcseconds, safely under the 4 arcsecond tolerance. 


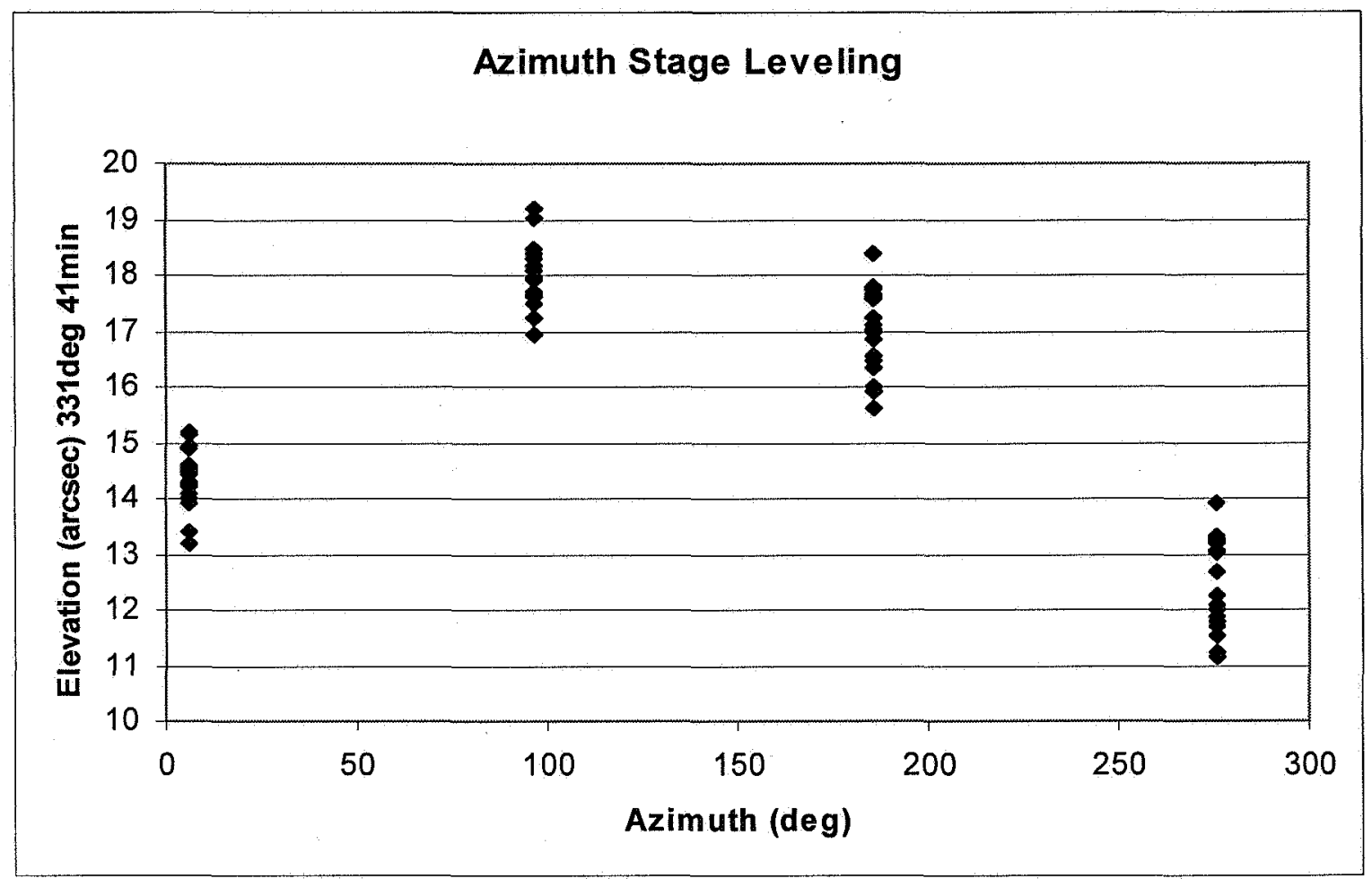

Figure 8. Results from Azimuth Stage Leveling

Using the adjustment and locking screws on the base plate to level the theoferometer to a position normal to the local gravity vector was difficult and approximating adjustment size was rough at best, limiting the ability to bring elevation readings under 4 arcseconds apart. Inherent in the prototype design are problems with mechanical drift in threaded parts and instabilities in the setup under vibration. Even with these faults in the design, the theoferometer can be aligned orthogonal to the local gravity vector using an external reference under a 4 arcsecond tolerance.

\section{B. Stage Orthogonality}

Examining and adjusting the orthogonality of the azimuth and elevation stages of the theoferometer centered on eliminating variations seen in azimuth readings as the theoferometer was scanned across several degrees in elevation. The orthogonality error appeared as unexpected angular displacement in the azimuth direction and was noticeable in the variation of azimuth readings as the elevation position was varied. Qualitatively, this runout error is a function of the travel distance of the stage and the sine of the angular displacement seen in the azimuth direction. To meet our goal of a sub-arcsecond measurement uncertainty for the theoferometer as a whole, the variation seen in the azimuth readings needed to be less than 1 arcsecond.

The stage orthogonality analysis used the corrected position readings, and the comparison between azimuth readings used the mean of the 10 shots taken at a given elevation. Adjustments to the angle between the azimuth and elevation stages were made with a micrometer mounted on the sine plate adjoining the two stages. Once the azimuth readings were within the 1 arcsecond limit, the micrometer was staked with epoxy to prevent the stages from settling out of their newly aligned position. The results of the final adjustment are shown in Figure 9. 


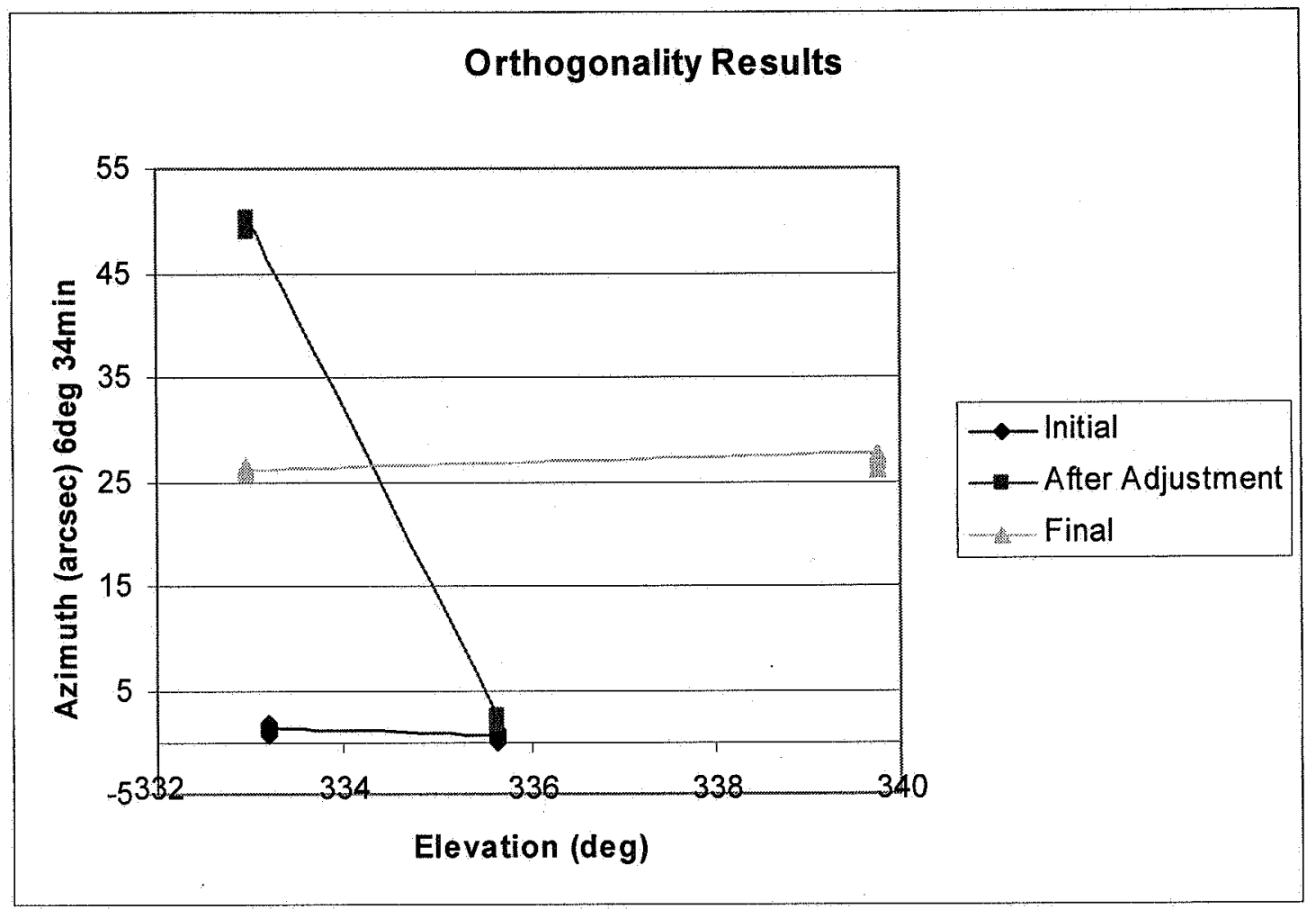

Figure 9. Results of Stage Orthogonality Testing

Figure 9 shows the angular displacement in the azimuth direction with variations in elevation. The initial azimuth displacement across $2.5^{\circ}$ of elevation change is less than 1 arcsecond. An adjustment of less than $1 \mu \mathrm{m}$ with the micrometer resulted in an azimuth displacement of 48.6 arcseconds, generating a significant error in the stage orthogonality. Reversing the original adjustment with the micrometer reduced the angular displacement seen in elevation to back under 1 arcsecond and within the acceptable error for stage orthogonality.

The results show the original mechanical design of the sine plate for stage orthogonality and the leveling procedure used during assembly to be accurate enough to maintain the sub-arcsecond measurement envelope of the theoferometer. The tolerance of less than 1 arcsecond azimuth variation was based on the available elevation range. The capability to adjust the orthogonality of the azimuth and elevation stages with a micrometer is too coarse to produce the sub-arcsecond changes possibly required to further reduce the measurement uncertainty of the theoferometer. The inability of the theoferometer and associated experimental setup to take data across a range of elevations greater than $6.5^{\circ}$ is insignificant in that metrology applications using the theoferometer will require less than $5^{\circ}$ of range in elevation.

\section{Software Sensitivity}

The software sensitivity analysis was based on a series of 25 shots with the theoferometer in a single position and the small angle generator set to 53 arcseconds azimuth and 17 arcseconds elevation. The variance of the azimuth software tilts was 0.18 arcseconds, and the variance of the elevation software tilts was 0.46 arcseconds. The difference in variances between the directions is potentially due to differences in the resolution of the CCD on the optical stage of the theoferometer.

\section{Characterization of Measurement Uncertainty}

The characterization of the measurement uncertainty of the theoferometer was critical to proving the capability of the instrument as a sub-arcsecond replacement for the theodolite. Figure 10 shows the results of the characterization of measurement uncertainty for the theoferometer. All of the mean values of data taken at each position of the small angle generator is contained within a \pm 0.5 arcsecond spread, with average errors in azimuth and elevation of 0.28 arcseconds in each direction. This demonstrated measurement uncertainty of the theoferometer in verifying tilts in a test surface is 0.3 arcseconds, under the 1 arcsecond goal for the prototype. 


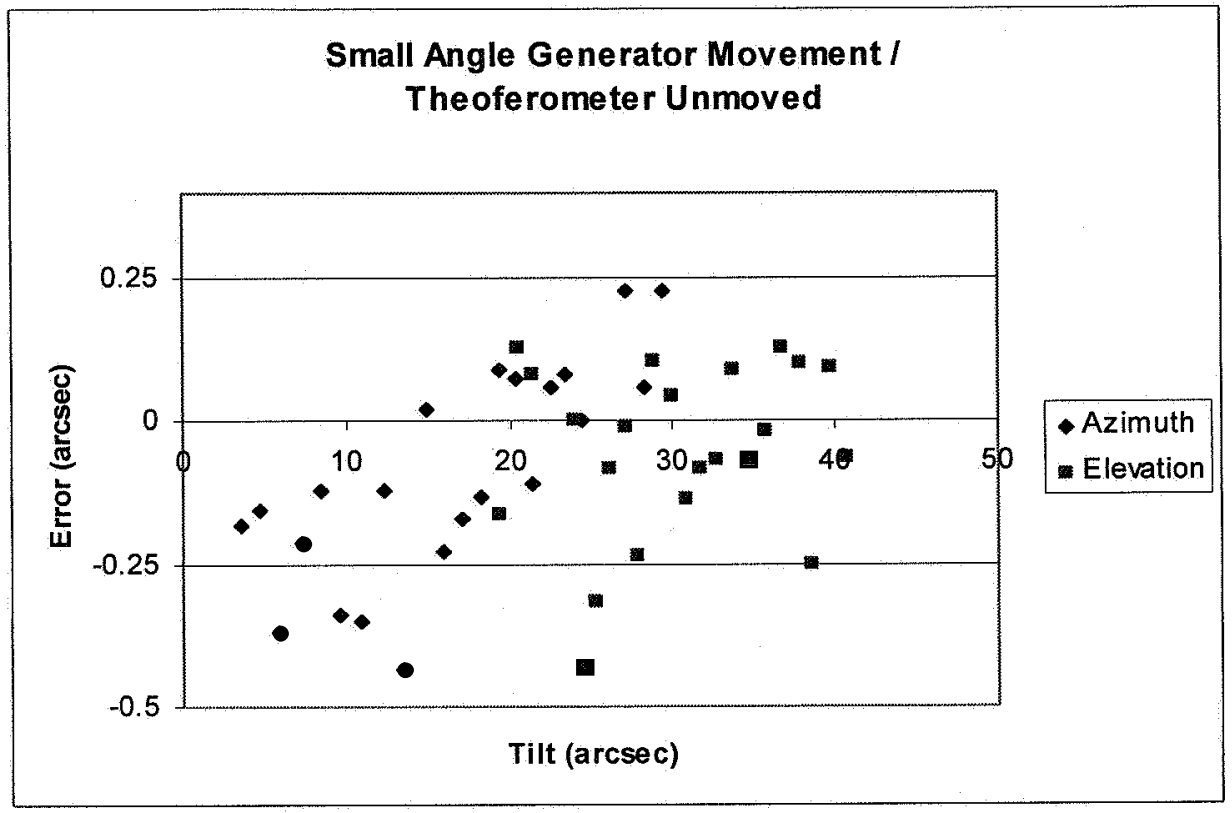

Figure 10. Results of Measurement Uncertainty Characterization

\section{Conclusion}

The concept of using interferometry with the theoferometer has been demonstrated to be an improvement over existing metrology instrumentation, reducing the measurement uncertainty in metrology and alignment applications to the sub-arcsecond level. The prototype theoferometer must be redesigned for increased portability if it is participate in full environment testing with flight hardware and move to replace the theodolite as the primary metrology tool. Problems with vibration isolation, stability, destabilization of the laser on the optical stage, and sensitivity to contrast and fringe density in the software will also need to be addressed in the redesign. Details of the optical, mechanical, and software redesign of the theoferometer and alignment and characterization of a second prototype will be discussed in a later paper. ${ }^{5}$

\section{Acknowledgments}

The author would like to thank the following people for their mentorship, advice, and support during this phase of the project: Ronald Toland at NASA/GSFC, Dr. Robert Sanner at the University of Maryland, Brad Frey, Doug Leviton, the Optics Branch at NASA/GSFC, and the Maryland Space Grant Consortium.

\section{References}

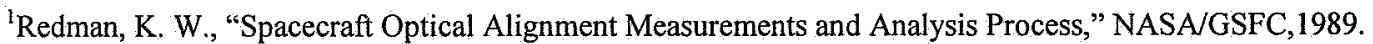

${ }^{2}$ Eichorn, W. L., "Optical Alignment Measurements at Goddard Space Flight Center," Applied Optics, Vol. 21, No. 21, 1 Nov. 1982.

${ }^{3}$ Toland, R. W., Leviton, D., Koterba, S., "Theoferometer for High Accuracy Optical Alignment and Metrology," SPIE Space Systems Engineering and Optical Alignment Mechanisms Conference, 5528B, Vol. 5528, SPIE, Denver, Colorado, 2004.

${ }^{4}$ Toland, R. W., "Absolute Measurement of Tilts via Fourier Analysis of Interferograms," SPIE Space Systems Engineering and Optical Alignment Mechanisms Conference, 5528B, Vol. 5528, SPIE, Denver, Colorado, 2004.

${ }^{5}$ Korzun, A. M., Toland, R. W., Ohl, R. G., Sanner, R., Holmes, V., Worrel, L., "Theoferometer for Construction of Precision Optomechanical Assemblies," SPIE Astronomical Telescopes and Instrumentation Conference, AS09, SPIE, Orlando, Florida,
} 2006. 